\title{
Uso e aceitação de Robôs, Inteligência Artificial e Automação dos Serviços em Hospitalidade e Turismo frente à Pandemia de COVID-19: uma proposta de pesquisa
}

\section{Use and acceptance of Robots, Artificial Intelligence and Automation of Services in Hospitality and Tourism in the face of the COVID-19 Pandemic: a research proposal}

\author{
Uso y aceptación de Robots, Inteligencia Artificial y Automatización de Servicios en \\ Hostelería y Turismo ante la Pandemia del COVID-19: una propuesta de investigación
}

\author{
Marilia Ferreira Paes-Cesário \\ Luiz Mendes Filho
}

Artigo Selecionado - Edição especial Inovação e Empreendedorismo em Turismo: interação entre os diferentes atores

\begin{abstract}
Resumo: Esse artigo teórico tem como objetivo propor um novo modelo com a junção de constructos da Teoria do Comportamento Planejado (Theory of Planned Behavior - TPB) e da Teoria Unificada de Aceitação e Uso da Tecnologia (Unified Theory of Acceptance and Use of Technology - UTAUT) para incentivar a realização de uma pesquisa empírica, na qual, através do modelo, seja possível observar se, a partir do maior acesso e disponibilidade de uso das tecnologias, impulsionado ainda mais agora, em virtude das necessidades trazidas pela Pandemia da COVID-19, haverá um aumento na intenção de uso e aceitação de tecnologias como robôs, inteligência artificial e automação dos serviços (RAISA - Robots, Artificial Inteligence and Self Automation) em hospitalidade. Foram escolhidos os seguintes constructos: Atitude (TPB), Normas Subjetivas (TPB), Expectativa de desempenho (UTAUT), Expectativa de esforço (UTAUT) e Condições Facilitadoras (UTAUT). A partir deles foram definidas hipóteses para serem validadas quanto à pesquisa empírica. Através de uma pesquisa bibliográfica, faz-se uma revisão sobre a evolução do uso das tecnologias no turismo e sobre o comportamento dos consumidores e das empresas, principalmente após a pandemia da COVID-19. Posteriormente, faz-se a proposição do novo modelo, através da aglutinação dos constructos e eliminação daqueles que acreditou-se serem capazes de trazer apenas respostas repetidas, por terem como foco as mesmas análises. Chega-se então às seis hipóteses, sobre o uso e a aceitação das tecnologias RAISA pelos turistas em suas próximas viagens, a serem validadas na pesquisa empírica, com o modelo proposto, através dos cinco constructos escolhidos.

Palavras-chave: Tecnologia; Turismo; RAISA; Theory of Planned Behavior; Unified Theory of Acceptance and Use of Technology.
\end{abstract}

\begin{abstract}
This theoretical article aims to propose a new model by joining constructs from the Theory of Planned Behavior (TPB) and the Unified Theory of Acceptance and Use of Technology (UTAUT) to encourage realization from an empirical research, in which through the model, it is possible to observe whether, from the greater access and availability of use of technologies, boosted even more now, due to the needs brought by the COVID-19 Pandemic, if there will be an increase in the intention of use and acceptance of technologies such as robots, artificial intelligence and service automation (RAISA - Robots, Artificial Intelligence and Self Automation) in hospitality. The following constructs were chosen: Attitude (TPB), Subjective Norms (TCP), Performance Expectation (UTAUT), Effort Expectation (UTAUT) and Facilitating Conditions (UTAUT) and from them hypotheses were defined to be validated during the empirical research. Through a bibliographical research, a review is made on the evolution of the use of technologies in tourism and on the behavior of consumers and companies, especially after the COVID-19 pandemic. Subsequently, the proposition of the new model is made, through the agglutination of constructs and elimination of those that were believed to be capable of bringing only repeated answers, as they focus on the same analyses. We then arrive at the six hypotheses, about the use and acceptance of RAISA technologies by tourists on their next trips, to be validated from the empirical research with the proposed model, through the five chosen constructs
\end{abstract}

${ }^{1}$ Formação/curso: Doutoranda em Turismo. Instituição: UNIVERSIDADE FEDERAL DO RIO GRANDE DO NORTE (UFRN), Natal RN, Brasil. E-mail: marilia.cesario@gmail.com

${ }^{2}$ Formação/curso: Doutor em Administração. Instituição: UNIVERSIDADE FEDERAL DO RIO GRANDE DO NORTE (UFRN), Natal RN, Brasil. E-mail: luiz.mendes@ufrn.br 


\section{ARTIFICIAL E AUTOMAÇÃO DOS SERVIÇOS \\ EM HOSPITALIDADE E TURISMO FRENTE À \\ PANDEMIA DE COVID-19: UMA PROPOSTA DE \\ PESQUISA}

Keywords: Technology; Tourism; RAISA; Theory of Planned Behavior; Unified Theory of Acceptance and Use of Technology.

Resumen: Este artículo teórico tiene como objetivo proponer un nuevo modelo mediante la unión de constructos de la Teoría del Comportamiento Planificado y la Teoría Unificada de Aceptación y Uso de Tecnología para incentivar la realización desde una investigación empírica, donde a través del modelo, es posible observar si, desde el mayor acceso y disponibilidad de uso de tecnologías, impulsado aún más ahora, debido a las necesidades que trajo la Pandemia COVID-19, habrá un incremento en la intención de uso y aceptación de tecnologías como robots, inteligencia artificial y automatización de servicios ( RAISA - Robots, Inteligencia Artificial y Auto Automatización) en hostelería. Se eligieron los siguientes constructos: Actitud (TCP), Normas subjetivas (TCP), Expectativa de desempeño (UTAUT), Expectativa de esfuerzo (UTAUT) y Condiciones facilitadoras (UTAUT) y a partir de ellas se definieron hipótesis para ser validadas durante la investigación empírica. A través de una investigación bibliográfica, se realiza una revisión sobre la evolución del uso de tecnologías en el turismo y sobre el comportamiento de consumidores y empresas, especialmente después de la pandemia COVID-19. Posteriormente, se realiza la propuesta del nuevo modelo, mediante la aglutinación de constructos y eliminación de aquellos que se creían capaces de traer solo respuestas repetidas, ya que se enfocan en los mismos análisis. Luego llegamos a las seis hipótesis, sobre el uso y aceptación de las tecnologías RAISA por parte de los turistas en sus próximos viajes, para ser validadas a partir de la investigación empírica con el modelo propuesto, a través de los cinco constructos elegidos.

Palabras Clave: Tecnología; Turismo; RAISA; Theory of Planned Behavior; Unified Theory of Acceptance and Use of Technology.

\section{Introdução}

Com o passar dos anos, o aumento do acesso e maior uso das tecnologias, o modo como as pessoas fazem suas compras ou usufruem de um serviço tem mudado de maneira surpreendente. Os consumidores atuais, em sua maioria, em algum momento dos seus comportamentos de compras; seja na pesquisa, efetivação da compra ou relato da experiência, têm usado as inovações tecnológicas como aliadas. E isso tem demandado, consequentemente, uma mudança de comportamento por parte das organizações, através da implantação de novos e automatizados processos, na maneira de ofertar o produto ou serviço ao consumidor e, principalmente, na maneira como as empresas têm se posicionado nestes mercados.

E, como consequência dessas mudanças, é perceptível que os serviços de turismo e hospitalidade não têm se comportado de maneira diferente. Afinal, além da questão das inovações tecnológicas, acrescenta-se ainda a chamada Economia da Experiência, que é um termo cunhado por Pine e Gilmore (1998) e representa uma maneira de atuar no mercado, na qual o mais importante é proporcionar a este

\footnotetext{
Revista Acadêmica Observatório de Inovação do Turismo, v. 15, n. 3, dezembro/2021
} 


\section{ARTIFICIAL E AUTOMAÇÃO DOS SERVIÇOS \\ EM HOSPITALIDADE E TURISMO FRENTE À \\ PANDEMIA DE COVID-19: UMA PROPOSTA DE \\ PESQUISA}

novo consumidor não apenas a venda de um produto ou serviço, mas a oportunidade de vivenciar uma experiência diferenciada e de qualidade com os produtos e serviços por ele escolhidos.

Sendo assim, verifica-se que a questão da inovação tecnológica já é um fato e que é preciso ter a percepção correta do mercado, conhecendo muito bem quem é o cliente potencial da empresa ou quais as suas necessidades, com a intenção de saber o que pode se encaixar no processo de oferta da experiência desse cliente, principalmente em localidades turísticas, as quais demandam um cuidado maior com a experiência do visitante, uma vez que a diferença cultural e linguística pode gerar alguns entraves, não gerando um serviço de qualidade.

Willems et al. (2017) afirmam que estas novas tecnologias disponíveis no mercado de varejo apresentam um papel estratégico de criar diferenciais competitivos, otimizando a experiência de compra do consumidor. Assim, já está posta uma nova maneira de vender produtos ou prestar alguns tipos de serviços através de inovações tecnológicas. É neste contexto que surgem as tecnologias chamadas RAISA (Robots, Artificial Inteligence and Self Automation), tal como mencionam Ivanov e Webster (2017) sobre empresas de diversos setores econômicos que usam tecnologias como robôs, inteligência artificial e automação dos serviços, para melhorar os processos de operação, otimizar seus custos, criar experiência para o cliente e expandir sua capacidade de atendimento.

A utilização desses serviços tecnológicos pode, muitas vezes, influenciar o comportamento do cliente, aumentar ou não a atração pela marca e ainda ampliar a satisfação dos compradores, fatores esses que podem trazer resultados positivos para as empresas, aumentando assim a sua competitividade no mercado. Porém, para Ivanov e Webster (2018) enquanto pesquisas sobre robôs de serviço no turismo já estão ganhando impulso, os aspectos econômicos da adoção destes ainda são bastante negligenciados, mostrando que é um campo ainda carente de um olhar mais atento.

Contudo, no último ano, surgiu uma crise sanitária que, embora muitos imaginassem a possibilidade de um dia acontecer ao mundo, pouca importância foi dada e pouco ou nenhum planejamento foi feito para se antecipar tal situação. Fala-se aqui sobre o que, de acordo com Hall, Scott e Gossling (2020) foi uma pneumonia de causa desconhecida detectada na cidade de Wuhan, na China, relatada pela primeira vez ao Escritório de Países da OMS (Organização Mundial de Saúde) em 31 de dezembro de 2019, e que deu início a essa crise sanitária de proporções não vistas nas últimas décadas e que, rapidamente, transformou-se na Pandemia da COVID-19. Essa doença, que vem desafiando todo o mundo e além do enorme impacto causado pela perda de inúmeras vidas, trouxe também diversos

Revista Acadêmica Observatório de Inovação do Turismo, v. 15, n. 3, dezembro/2021 


\section{ARTIFICIAL E AUTOMAÇÃO DOS SERVIÇOS \\ EM HOSPITALIDADE E TURISMO FRENTE À \\ PANDEMIA DE COVID-19: UMA PROPOSTA DE \\ PESQUISA}

problemas, entraves e modificações no que se refere à maneira como as relações sociais, comerciais e de deslocamento aconteciam entre os países, abalando, assim, diversos setores da economia.

Neste contexto, destaca-se a atividade turística, que foi uma das áreas mais impactadas, principalmente, por causa da forma de contágio da doença, uma vez que, para evitar a propagação, gerou a necessidade rígida de isolamento social e o fechamento de fronteiras. Após mais de um ano de enfrentamento da pandemia, muita coisa já mudou e é possível compreender que tudo será ainda mais diferente daqui para frente, não apenas referente às questões de protocolos de higiene que já são necessários, mas principalmente quanto à maneira de ofertar os serviços para esse consumidor que está mais conectado, mais informado e, agora, mais preocupado com riscos e questões referentes ao contágio de doenças.

Assim, é fato que, rapidamente as empresas precisaram adaptar-se a esse novo perfil ou então perderiam seus clientes para a concorrência. Neste processo de inovação e de diferentes maneiras de oferecer um serviço, acelerado pela pandemia da COVID-19, o que se vê surgir, de uma maneira muito forte, são as chamadas TAA (Tecnologias de Autoatendimento), que de acordo com Meuter et al. (2000) tem possibilitado a criação de interfaces que habilitam os clientes a obter um serviço de fácil utilização, independente do envolvimento de funcionários das lojas físicas.

Independente da etapa do processo, se para compra do produto/serviço completo, apenas para realizar o pagamento final, ou ainda para comprar através de um aplicativo, o que se vê é uma aceleração na corrida dos estabelecimentos por implantar sistemas automatizados, online ou em lojas físicas, mas que possam proporcionar ao cliente uma experiência de compra mais rápida, de qualidade e, se possível, através de um ambiente de contato mais controlado. Essa realidade de comportamento nas empresas é facilmente encontrada na, ainda tímida, retomada das atividades turísticas em alguns lugares, na qual a tecnologia tem entrado de maneira mais forte, proporcionando a automação de serviços e processos que antes necessitavam de uma ou mais pessoas para realizá-los.

Contudo, para Parveza e Cobanoglu (2021) a pandemia da COVID-19 é prevista para ser uma crise de longo prazo, na qual além da perda econômica, o turismo também enfrenta uma crise de imagem afetando futuras carreiras na área. É preciso lidar com a complexidade dos ecossistemas turísticos e de proteção contra vírus e assim a cadeia produtiva do turismo pode decidir empregar robôs de serviço para um futuro melhor.

Sendo assim, é de suma importância fazer uma análise mais cuidadosa, principalmente pelo fato de que nem todos os consumidores têm essa gana de usar as tecnologias como dispositivos eletrônicos,

Revista Acadêmica Observatório de Inovação do Turismo, v. 15, n. 3, dezembro/2021 


\section{ARTIFICIAL E AUTOMAÇÃO DOS SERVIÇOS \\ EM HOSPITALIDADE E TURISMO FRENTE À \\ PANDEMIA DE COVID-19: UMA PROPOSTA DE \\ PESQUISA}

aplicativos ou interagir com robôs, e muitos também não possuem ainda habilidade suficiente para, ao usar esses novos canais de atendimento, serem capazes de usufruir de uma boa experiência, sem complicações ou entraves. E ainda, pelo fato de que essa nova modalidade de oferta dos serviços pode, como uma consequência negativa, gerar certa repulsa e complicar ou inviabilizar a concretização da compra por parte de alguns usuários que podem ter dificuldade com as tecnologias ou até mesmo certo receio em tentar usar essas inovações.

Considerando o exposto e pensando no consumidor, na forma como ele recebe essas inovações e, principalmente, se está disposto a usufruir de tais mudanças, torna-se interessante fazer os seguintes questionamentos: é possível, ao integrar os constructos mais relevantes de duas teorias, propor hipóteses que possam ser testadas, em uma pesquisa empírica futura, para entender melhor o comportamento dos consumidores em relação ao uso dessas novas tecnologias?

Desta maneira, esse artigo tem como objetivo principal compor um novo modelo de pesquisa, a partir de constructos da Teoria do Comportamento Planejado (TCP), do inglês Theory of Planned Behavior (TPB), e da Teoria Unificada de Aceitação e Uso da Tecnologia, do inglês Unified Theory of Acceptance and Use of Technology (UTAUT), propondo hipóteses a serem validadas em uma pesquisa empírica futura para poder confirmar ou não a aceitação e uso das tecnologias RAISA por parte dos potenciais turistas e como se daria a experiência deles.

Como metodologia para a concretização dessa proposta é realizada uma pesquisa bibliográfica em artigos que tratem do uso das tecnologias pelo turismo, do advento da pandemia da COVID-19 e suas implicações e das teorias mencionadas, para que ao fazer um apanhado de seus conceitos e constructos trabalhados, seja possível levantar as hipóteses de estudo.

\section{Tecnologias, Turismo e o Cenário da COVID-19}

É importante observar que o uso e acesso às tecnologias já vinha crescendo antes do surgimento da pandemia da COVID-19. Muito embora o acesso e a utilização de cada um dos tipos de tecnologias possam variar bastante, o mercado turístico usufrui hoje de diversos processos que estão totalmente inseridos nas inovações tecnológicas em alguns lugares do mundo. Além disso, há o fato de que existem inúmeros benefícios na adoção de tecnologias, tais como a RAISA (Robots, Artificial Inteligence and Self Automation), sendo um dos fatores mais mencionados pelos empresários que adotam esses recursos, a questão financeira.

Revista Acadêmica Observatório de Inovação do Turismo, v. 15, n. 3, dezembro/2021 


\section{ARTIFICIAL E AUTOMAÇÃO DOS SERVIÇOS \\ EM HOSPITALIDADE E TURISMO FRENTE À \\ PANDEMIA DE COVID-19: UMA PROPOSTA DE \\ PESQUISA}

De acordo com Ivanov e Webster (2017), há uma redução nos custos trabalhistas quando adotam a RAISA, ao passo que robôs de serviço, chatbots e quiosques de autoatendimento podem operar 24 horas por dia, 7 dias por semana, muito mais do que a carga horária habitual de 40 horas semanais de um funcionário. Além disso, os chatbots podem servir inúmeros clientes simultaneamente, o que não é a norma com funcionários humanos.

Desta forma, embora tenha demorado a chegar ao turismo, esse uso maior das tecnologias de automação de serviços já é uma realidade bastante conhecida em algumas partes do mundo. Um exemplo disso é o uso de check-in online, quiosques de auto check-in, portões automatizados de controle de fronteira com leitores de passaporte/cartão de identificação biométricos e embarque móvel dos passes já disponíveis em muitos aeroportos e máquinas de venda automática de alimentos e bebidas que estão por toda parte (HEINEKE et al., 2017).

Para Zeng, Chen e Lew (2020), embora os robôs tenham desempenhado um papel essencial em ambientes industriais e empregos de alto risco por muitos anos, pouca atenção tem sido dada aos papéis que os robôs poderiam desempenhar em ambientes interativos "de alto toque" ou "alta relação interpessoal", como o setor de hospitalidade. E essa é uma preocupação pertinente, uma vez que por mais que exista automação nos serviços e vendas de produtos, sempre existirão consumidores que vão preferir o contato humano ou até mesmo aqueles que usarão as máquinas. Entretanto, quando tiverem a oportunidade de contato com pessoas, irão ter uma expectativa muito elevada quanto à qualidade do atendimento.

Corroborando com essa pouca atenção que era dada ao uso dessas tecnologias, de acordo com Ivanov et al. (2019), a incorporação da robótica chegou relativamente atrasada às indústrias das viagens, turismo e hospitalidade. Isso provavelmente porque muitos dos serviços prestados exigem sofisticadas reações às necessidades do cliente, o que muitas vezes uma máquina ainda não pode fazer. Não apenas esse, mas outros entraves, como a questão da pouca e até mesmo da não disponibilidade das estruturas necessárias para a existência dessas tecnologias, tais como conexão à internet em diversos espaços, acesso aos equipamentos como smartphones ou tablets fazem com que inúmeras localidades ainda não possam usufruir de todos esses benefícios tecnológicos. Contudo, a pesquisa sobre robôs em viagens, turismo e hospitalidade está ganhando força e pode-se esperar um aumento significativo no futuro, em linha com a adoção real de robôs por empresas turísticas (IVANOV et al., 2019).

Somando-se a tudo isso, a chegada inesperada de uma crise sanitária de proporções mundiais, impedindo a circulação de pessoas, fez com que várias tecnologias, que ainda não tinham sido colocadas

Revista Acadêmica Observatório de Inovação do Turismo, v. 15, n. 3, dezembro/2021 


\section{ARTIFICIAL E AUTOMAÇÃO DOS SERVIÇOS \\ EM HOSPITALIDADE E TURISMO FRENTE À \\ PANDEMIA DE COVID-19: UMA PROPOSTA DE \\ PESQUISA}

tanto à prova pelas empresas, tais como aplicativos de compras online, uso de sistemas de delivery ou takeaway, quiosques de autoatendimento, passassem a ser a única solução para conseguir continuar no mercado e vender para seus clientes, que precisavam adquirir certos produtos e serviços, mas não podiam ou não queriam se expor.

Com o aumento do uso das tecnologias, foi visto que durante a pandemia da COVID-19, a vantagem dos robôs em ajudar a manter a distância social também tem sido mostrada ao público. Robôs podem realizar tarefas relacionadas à logística, desinfecção e fornecer informações da COVID-19 às pessoas, reduzindo a ameaça de infecção (ZENG; CHEN; LEW, 2020). É crescente o acesso dessas tecnologias por novos grupos de pessoas, pois de acordo com Botelho, Cardoso e Canella (2020) em relação ao momento atual, acredita-se que, no processo de atração e fidelização de clientes que não usavam plataformas de delivery regularmente, estejam sendo alcançados públicos com perfis diferentes dos habituais.

Isso está ocorrendo inclusive com as pessoas mais velhas, que são grupo de risco para a COVID19 e que, em sua maioria, não costumavam usar tais aplicativos. Agora, pela primeira vez, podem experimentar comprar comida online, por exemplo. E assim, ainda de acordo com os autores, como consequência da pandemia, bares, lanchonetes, restaurantes e congêneres adotaram ou intensificaram sua presença no ambiente digital, principalmente em aplicativos de delivery de comida (BOTELHO; CARDOSO; CANELLA, 2020).

Logo, em decorrência das necessidades impostas pela pandemia, os serviços diretos ao consumidor aumentaram rapidamente, enquanto muitas marcas tradicionais de supermercado tentam desenvolver uma rota fácil para o mercado e se tornar as primeiras marcas online. Assim, esse tipo de comércio de alimentos tem testemunhado um aumento explosivo na demanda (GALANAKIS et al., 2021). Por essa razão, é possível perceber o aumento do uso das tecnologias pelos consumidores, muitas vezes, por uma questão de necessidade.

Da mesma maneira, esse impacto está sendo percebido na indústria do turismo, que consequentemente tem demandado um maior uso das tecnologias com a intenção de sobreviver à crise. Como abordam Hall, Scott e Gossling (2020) a pandemia não é apenas uma crise de saúde de imensa proporção, mas é também uma reestruturação iminente da ordem econômica global, como resultado do impacto econômico da supressão do vírus, potencialmente incluindo a natureza do turismo internacional como fenômeno econômico e social. Desta forma, surge quase imediatamente o questionamento se, de

Revista Acadêmica Observatório de Inovação do Turismo, v. 15, n. 3, dezembro/2021 
ARTIFICIAL E AUTOMAÇÃO DOS SERVIÇOS

EM HOSPITALIDADE E TURISMO FRENTE À

PANDEMIA DE COVID-19: UMA PROPOSTA DE

PESQUISA

fato, os consumidores estão ou estarão dispostos e preparados para usufruir dessas tecnologias também na atividade turística, de uma maneira geral.

Portanto, para tentar entender o comportamento dos turistas em relação ao uso dessas tecnologias, faz-se uso da Teoria do Comportamento Planejado e da Teoria Unificada de Aceitação e Uso da Tecnologia, com a intenção de criar um modelo de pesquisa que possa identificar o comportamento dos consumidores nas suas viagens após a pandemia, uma vez que a mesma já proporcionou, como relatado, um aumento de acesso e uso das tecnologias.

\section{Abordagens sobre a Teoria do Comportamento Planejado e a Teoria Unificada de Aceitação e Uso da Tecnologia}

Com o intuito de investigar as intenções de comportamento dos turistas sobre o uso e aceitação das tecnologias, tais como as já mencionadas RAISA, em suas viagens após a pandemia da COVID-19, faz-se aqui uma revisão da literatura no que se refere às duas teorias (TCP e UTAUT) que, acredita-se, ao serem combinadas, podem servir de base para uma análise mais precisa. Consequentemente, é possível dimensionar em uma pesquisa empírica se há influência do uso dessas tecnologias no processo de tomada de decisão de compra pelo turista, quando este fizer uso dos serviços de tecnologias RAISA disponíveis em suas viagens.

\subsection{Teoria do Comportamento Planejado (TCP)}

Como base fundamental para analisar o comportamento de compra dos consumidores sobre o uso e aceitação das tecnologias, é imprescindível apresentar a Teoria do Comportamento Planejado (TCP), quando Ajzen (1991) tenta entender o comportamento humano de uma forma geral, a partir da intenção comportamental e de variáveis explicativas. Assim, Ajzen traz o seu primeiro conceito, a partir do qual inúmeros estudos foram elaborados, inclusive por ele mesmo posteriormente (SILVEIRA, 2016).

Essa teoria parte da tentativa de contornar o entrave existente em outras teorias para tentar explicar o comportamento humano. É, portanto, uma extensão da teoria do TRA (Theory of Reasoned Action) e foi necessário fazer tal ação pelas limitações do modelo original em lidar com comportamentos sobre o qual as pessoas têm controle volitivo incompleto, ou seja, não possuem total controle sobre a

Revista Acadêmica Observatório de Inovação do Turismo, v. 15, n. 3, dezembro/2021 


\section{ARTIFICIAL E AUUTOMAÇÃO DOS SERVIÇOS \\ EM HOSPITALIDADE E TURISMO FRENTE À \\ PANDEMIA DE COVID-19: UMA PROPOSTA DE \\ PESQUISA}

sua decisão de comportamento (AJZEN, 1991). Assim, a TCP utiliza a base teórica da TRA proposta por Ajzen e Fishbein (1980), e ainda incorpora o conceito do controle comportamental percebido, criado por Ajzen, em 1985.

Desta maneira, a TCP tem como objetivo principal compreender e predizer os comportamentos sociais, isto é, busca prever as intenções comportamentais que precedem e que condicionam os comportamentos reais, em vez de conhecer unicamente as atitudes, as normas subjetivas e as crenças do indivíduo, como fazia anteriormente o TRA (PINTO, 2007). E, para este estudo, é mais interessante que a teoria utilizada seja a TCP, uma vez que de acordo com Matos e Sardinha (1999) a Teoria da Ação Racional tinha claras limitações no que se refere à relação com comportamentos em que a pessoa não tem controle completo sobre a própria vontade, ou seja, em situações em que a decisão sobre um comportamento é influenciada por algum grau de oportunidade ou recurso.

E isso se justifica, no caso específico do uso das tecnologias RAISA (oportunidade ou recurso), por turistas de outras localidades, uma vez que o uso dessas tecnologias a serviço do cliente pode ser um recurso diferencial que o influencie a utilizá-lo, de forma a facilitar sua estada na localidade, principalmente frente às barreiras linguísticas. Ou mais especificamente, por causa do distanciamento necessário imposto pela pandemia da COVID-19.

Segundo a TCP, as intenções de realizar comportamentos de diferentes tipos podem ser previstas com alta precisão pelas atitudes em relação ao comportamento, normas subjetivas e percepção de controle comportamental. Essas intenções, juntamente com as percepções de controle de comportamento, são responsáveis por uma variação considerável no comportamento real (AJZEN, 1991). De acordo com Armitage e Christian (2003) vale afirmar que a TCP é, hoje, o modelo dominante das relações atitude-comportamento, possibilitando, assim, a previsão acurada das intenções comportamentais a partir de um conjunto reduzido de variáveis antecedentes - atitude relativa ao comportamento, norma subjetiva e controle percebido do comportamento.

Considerando que a intenção é peça-chave no direcionamento do comportamento, a teoria do Ajzen (1991) a define como: Intenção: capturar os fatores motivacionais que influenciam um comportamento; são indicações de como as pessoas estão dispostas a tentar, ou seja, de quanto esforço estão planejando exercer, a fim de realizar o comportamento. Para entender melhor esta teoria, é importante observar na Figura 1 o modelo proposto por Ajzen (1991).

Revista Acadêmica Observatório de Inovação do Turismo, v. 15, n. 3, dezembro/2021 
Figura 1 - Teoria do Comportamento Planejado

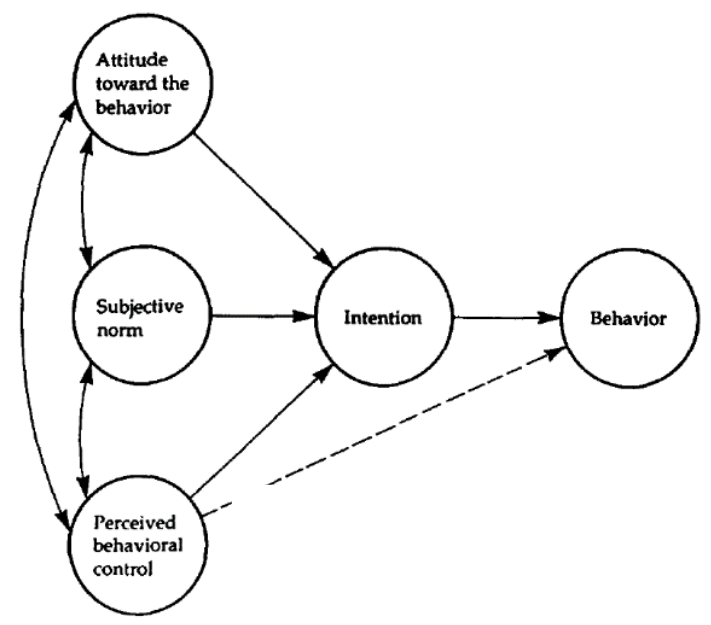

Fonte: Ajzen (1991).

Desta maneira, percebe-se que de acordo com Ajzen (1991), a TCP possui três determinantes conceituais de intenção, que por fim, interferem no comportamento. São eles: Atitude: grau em que uma pessoa tem uma avaliação favorável ou desfavorável do comportamento em questão. Normas subjetivas: refere-se à pressão social percebida para realizar ou não o comportamento. Controle do Comportamento Percebido: refere-se à facilidade ou dificuldade percebida de executar o comportamento e presume-se que reflita a experiência passada bem como impedimentos e obstáculos antecipados.

Como regra geral, quanto mais favorável for a atitude e a norma subjetiva com relação a um comportamento e quanto maior for o controle comportamental percebido, mais forte deve ser a intenção de um indivíduo de realizar o comportamento em consideração (AJZEN, 1991). Assim, com a intenção de tornar mais robusta a avaliação da intenção dos turistas de usar as tecnologias RAISA em suas viagens, faz-se agora uma revisão sobre a segunda teoria a ser utilizada, que é a Teoria Unificada de Aceitação e Uso da Tecnologia.

\subsection{Teoria Unificada de Aceitação e Uso da Tecnologia}

Fazendo um contraponto com a importância da TCP para analisar a intenção dos turistas de usar tecnologias nas destinações, vale a pena entender a relação dessa teoria já mencionada com a Teoria 


\section{ARTIFICIAL E AUTOMAÇÃO DOS SERVIÇOS \\ EM HOSPITALIDADE E TURISMO FRENTE À \\ PANDEMIA DE COVID-19: UMA PROPOSTA DE \\ PESQUISA}

Unificada de Aceitação e Uso da Tecnologia (Unified Theory of Acceptance and Use of Technology UTAUT). A relevância de fazer a integração das teorias é que não adiantaria o turista ter à sua disposição todas as tecnologias mencionadas ou ainda, ter predisposição de usar tal facilidade (comportamentos observados pela TCP), mas, ser impedido por uma não aceitação do uso da tecnologia em questão, seja por desconhecimento, medo ou qualquer outro motivo (comportamentos observados pela UTAUT).

Logo, este estudo traz a conceituação da UTAUT, que de acordo com algumas pesquisas realizadas (VENKATESH et al., 2003; CARLSSON et al., 2006; PIKKARAINEN et al., 2004; BOBSIN et al., 2009) mostra-se como uma das teorias mais eficientes para avaliar a aceitação de uso das tecnologias. Assim, através de alguns dos seus constructos associados aos selecionados da teoria do TCP, acredita-se que seja possível propor hipóteses que possam ser verificadas com relação à disponibilidade de aceitação e uso das tecnologias RAISA.

Para entender melhor, faz-se aqui uma pequena reflexão sobre a origem dessa teoria, que atua como uma ferramenta útil na análise do sucesso da implementação de um SI ou na identificação da intenção de uso dos mesmos (VENKATESH et al., 2003), ganhando uma maior profundidade na compreensão do comportamento de uso dos indivíduos, o que agrega valor à tomada de decisão. $\mathrm{Na}$ literatura há vários modelos teóricos desenvolvidos, principalmente a partir de teorias em Psicologia e Sociologia, que são utilizados para explicar a aceitação da tecnologia e uso desta. Sendo assim, com a intenção de melhor explicar a aceitação de uso das tecnologias ao realizarem novas viagens, acredita-se que analisar uma outra teoria de uso da tecnologia pode elucidar mais ainda o comportamento do consumidor desses estabelecimentos turísticos, principalmente pelo fato desta última teoria ser um modelo já unificado. Assim, é interessante uma observação da UTAUT, que na realidade é uma revisão e síntese de outras oito teorias e modelos de usos da tecnologia.

As origens dessa teoria vêm, de acordo com Bobsin, Visentini e Rech (2009), quando Venkatesh e outros autores em 2003 criaram um modelo unificado de aceitação de tecnologia que incorpora elementos desses outros modelos. Para que fique mais bem explicado, vale ressaltar que os modelos utilizados foram: a Teoria da Ação Racional (TRA), proposta por Fishbein e Ajzen (1975); o Modelo de Aceitação da Tecnologia (TAM), de Davis (1989); o Modelo Motivacional (MM), de Vallerand (1997); a Teoria do Comportamento Planejado (TPB), de Ajzen (1991); o Modelo Combinado TAMTPB, proposto por Taylor e Tood (1995); o Modelo de Utilização do PC (MPCU), de Thompson, Higgins (1991); a Teoria da Difusão da Inovação, de Rogers (1995), aplicada em SI por Moore e

Revista Acadêmica Observatório de Inovação do Turismo, v. 15, n. 3, dezembro/2021 
Benbasat (1996); e a Teoria Social Cognitiva, de Bandura (1986), que foi ampliada para o contexto de uso de computadores por Compeau e Higgins (1995).

Desta maneira, os idealizadores do modelo UTAUT propuseram quatro construtos: expectativa de desempenho, expectativa de esforço, influência social e condições facilitadoras. Estes, são moderados pela idade, experiência, gênero ou voluntariedade de uso e teriam influência direta sobre a intenção de uso e o uso efetivo da tecnologia (VENKATESH et al., 2003). Uma informação bastante relevante é trazida por Davis, Bagozzi e Warshaw (1989) quando destacam que para se explicar e incrementar a aceitação do usuário de TI é necessário, antes de tudo, desvendar o porquê de as pessoas aceitarem ou rejeitarem os computadores ou as tecnologias.

Ou seja, é necessário tentar entender que barreiras existem frente ao uso dessas novas tecnologias e o porquê de alguns indivíduos não conseguirem ou não terem a intenção de entender como seria o uso das mesmas, para então usufruírem dos serviços oferecidos. Abaixo, na Figura 2, é possível ver o modelo criado por Venkatesh et al. (2003):

Figura 2 - Modelo UTAUT

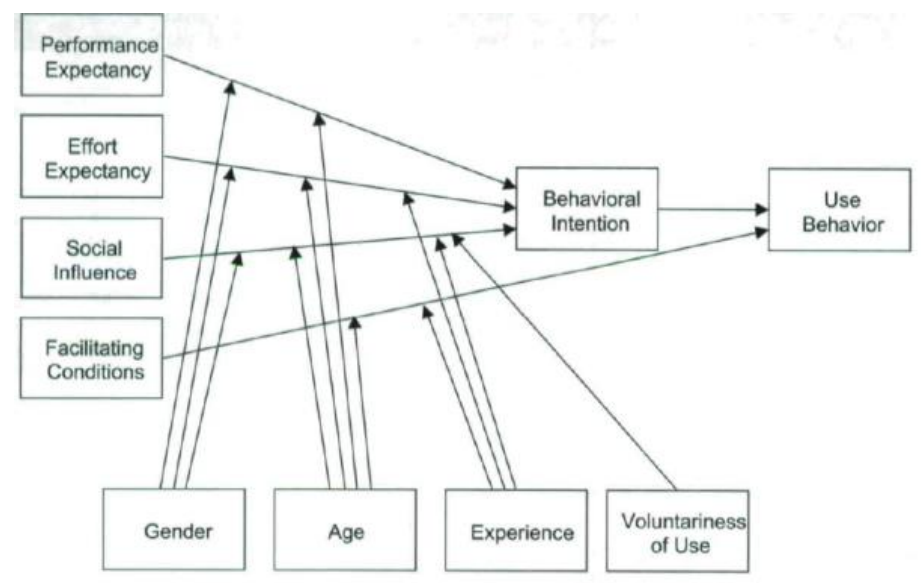

Fonte: Venkatesh et al. (2003)

Assim, destacam-se aqui os principais constructos trabalhados no UTAUT (VENKATESH et al., 2003), que são: Expectativa de Desempenho: grau em que um indivíduo acredita que o uso do sistema vai ajudá-lo a atingir ganhos no resultado do trabalho. Moderadores que influenciam: gênero e idade; Expectativa de Esforço: grau de facilidade associado ao uso do sistema. Moderadores que 


\section{ARTIFICIAL E AUTOMAÇÃO DOS SERVIÇOS \\ EM HOSPITALIDADE E TURISMO FRENTE À \\ PANDEMIA DE COVID-19: UMA PROPOSTA DE \\ PESQUISA}

influenciam: gênero, idade e experiência; Influência Social: grau em que um indivíduo percebe que outras pessoas importantes acreditam que ele deveria usar o novo sistema. Moderadores que influenciam: gênero, idade, experiência e voluntariedade de uso; Condições Facilitadoras: grau em que um indivíduo acredita que existe uma infraestrutura organizacional e técnica para suportar o uso do sistema. Moderadores que influenciam: idade e experiência.

Para justificar ainda mais a importância do seu uso, foram considerados alguns estudos que estão apresentados na descrição dos constructos utilizados no novo modelo, nos quais o modelo UTAUT tem se mostrado adequado para avaliação da possibilidade de sucesso no processo de implantação da tecnologia. Acredita-se que, ao fazer uma revisão dessas duas teorias - TCP e UTAUT, seja possível elencar constructos essenciais que possam compor um modelo de pesquisa a ser proposto para confirmar ou não a aceitação de uso das tecnologias RAISA por parte dos turistas.

Considerando que existem diferentes estudos conduzidos por autores de várias áreas que parecem tentar explicar os mesmos tipos de fenômenos, surgiu o interesse em conduzir uma discussão de cunho teórico no sentido de relacionar ou buscar identificar as interfaces entre a TCP e a UTAUT, visando à proposição de pesquisas que possam contribuir para o entendimento do comportamento do turista em destinações que possuam tecnologias RAISA à disposição deles.

Como um reforço à intenção de criação desse modelo de pesquisa, vale ressaltar o que foi citado por Venkatesh, Thong e Xu (2012) quando mencionaram que era recomendado que a teoria UTAUT fosse aplicada em outros contextos e com outras tecnologias distintas das que estudaram, com vistas a verificar a robustez da teoria. E foi com esse propósito que estes mesmos autores ampliaram esse modelo e desenvolveram a UTAUT2, em 2012, para ser aplicada em contextos específicos de consumo e que possui além desses quatro constructos do UTAUT outros três, que são: motivação hedônica, valor percebido e hábito (VENKATESH; THONG; XU, 2012).

Contudo, o presente estudo optou por usar o modelo UTAUT, com menos constructos, seguindo o princípio da parcimônia e gerando, assim, um número menor de hipóteses sem perder o propósito principal que é o de avaliar o uso e aceitação das novas tecnologias. De acordo com Venkatesh, Thong e Xu (2012), desde sua publicação original, o UTAUT tem servido como um modelo de linha de base e tem sido aplicado ao estudo de uma variedade de tecnologias, tanto organizacionais quanto nãoorganizacionais.

Assim, com o modelo formado pela integração dessas duas teorias, pretende-se identificar os fatores que influenciam direta ou indiretamente a aceitação e a intenção de uso das tecnologias e, em

Revista Acadêmica Observatório de Inovação do Turismo, v. 15, n. 3, dezembro/2021 
um segundo momento, o que pode ser feito utilizando esse modelo para gerar maiores trocas de informação e novos benefícios, através de uma pesquisa empírica.

\section{Modelo Proposto de Integração das Teorias TCP e UTAUT}

Muito embora as teorias estudadas neste estudo sejam, isoladamente, bastante úteis para realizar inúmeras avaliações de comportamento dos indivíduos em diversas áreas e situações, e já tenham sido utilizadas e validadas em diversos estudos de forma eficiente, foi sentida a necessidade de somar a capacidade que a TCP tem em analisar ações de comportamento em diversos momentos, com as habilidades específicas da UTAUT em observar as intenções de uso e aceitação das tecnologias, de forma a propor um modelo integrado entre as mesmas.

Embora considerando que a UTAUT é uma junção de elementos de várias outras teorias e que com isso houve um maior uso para as pesquisas, além do fato de ser uma das teorias mais apropriadas para responder sobre o uso das tecnologias, cogitou-se a junção dela com a TCP, uma vez que com a combinação das duas é possível avaliar não apenas a intenção do comportamento, mas também predisposição do uso das tecnologias. Contudo, ao avaliar essa integração das teorias, foi percebido que alguns constructos se repetem quanto à finalidade de resposta. Assim, aglutinou-se os dois modelos e exclui-se aqueles constructos que, de alguma maneira, se sobrepunham como semelhantes.

Para isso, da teoria do TCP foi excluído o constructo Controle do Comportamento Percebido, uma vez que o constructo Expectativa de Esforço do UTAUT também avalia o grau de facilidade associado ao uso do sistema e tem a experiência, como um dos moderados, igualmente ao constructo do Controle do Comportamento Percebido. Contudo, tal contructo faz uma análise ainda mais completa, especialmente ao avaliar moderadores também de gênero e idade. O outro constructo excluído, a Influência Social da teoria UTAUT, foi devido às Normas Subjetivas da TCP terem a mesma finalidade de resposta, o que ficaria repetitivo.

Portanto, no modelo proposto, há dois constructos do TCP, que são Atitude e Normas Subjetivas, três constructos do UTAUT, que são Expectativa de Desempenho, Expectativa de Esforço e Condições Facilitadoras e dois constructos que fazem parte de TCP e UTAUT ao mesmo tempo, que são Intenção e Comportamento de Uso. A proposta é tornar um modelo mais adequado para responder às questões quanto ao comportamento dos turistas frente à aceitação e ao uso das tecnologias RAISA em destinações turísticas.

Revista Acadêmica Observatório de Inovação do Turismo, v. 15, n. 3, dezembro/2021 
ARTIFICIAL E AUTOMAÇÃO DOS SERVIÇOS

EM HOSPITALIDADE E TURISMO FRENTE À

PANDEMIA DE COVID-19: UMA PROPOSTA DE

PESQUISA

Assim, após chegar a esse modelo proposto, faz-se uma análise conceitual de cada constructo, através de pesquisa bibliográfica, que o compõe e avalia-se também os seus usos em outros estudos para, a partir daí, propor as hipóteses de pesquisa.

Figura 3 - Modelo Proposto

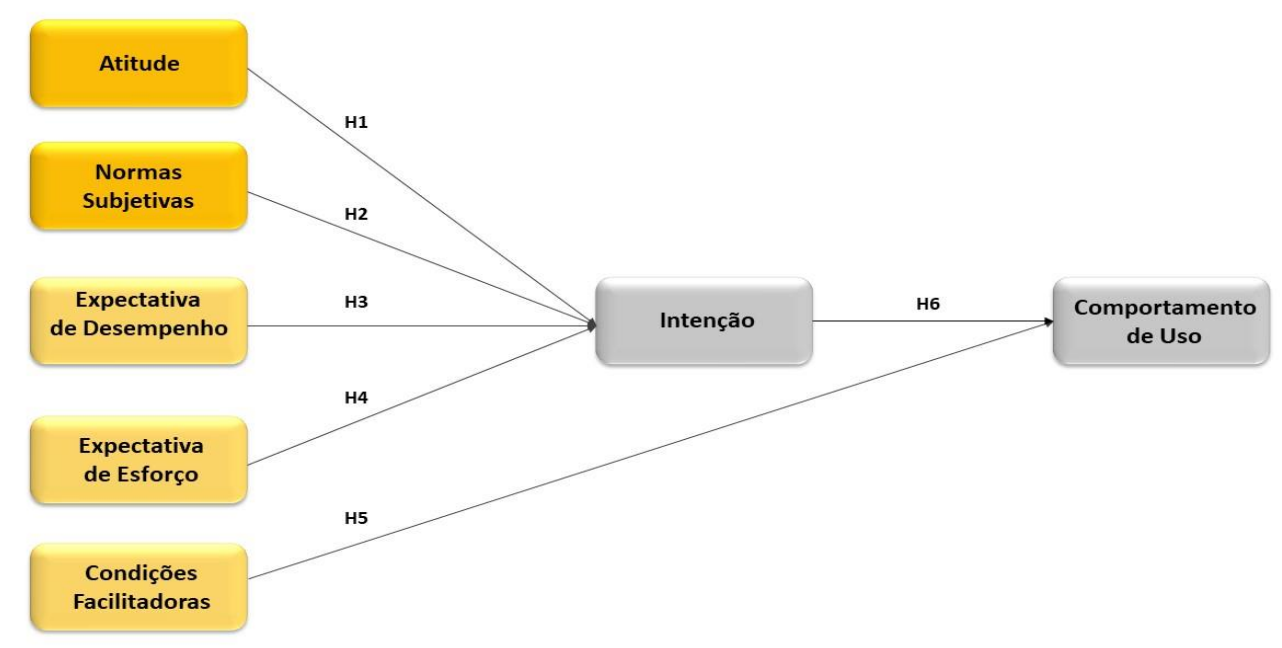

Fonte: Autores da pesquisa (2021).

\subsection{Atitude (TCP)}

Vindo da TCP, a atitude é o grau que uma pessoa tem uma avaliação favorável ou não favorável do comportamento em questão. Sendo assim, de acordo com Armitage e Conner (2001 apud MENDESFILHO; TAN; MILLS, 2012) quanto mais favorável for a atitude em relação ao comportamento, mais forte deve ser a intenção do indivíduo em realizá-lo. Para Rodrigues, Assmar e Jablonski (2000) as atitudes envolvem o que as pessoas pensam, sentem e como elas gostariam de se comportar em relação a um objeto atitudinal.

Desta maneira, provavelmente uma atitude favorável em relação ao uso das tecnologias incentivará os turistas a usá-las ao encontrar tais recurso nas destinações visitadas. Foi visto que a literatura existente e a pesquisada demonstra um impacto positivo da atitude frente às intenções comportamentais, tal como em Silveira (2016), que ao estudar o uso da bicicleta sob os fundamentos da Teoria do Comportamento Planejado, enfatiza o papel crucial da percepção de barreiras ao uso do 
ARTIFICIAL E AUTOMAÇÃO DOS SERVIÇOS

EM HOSPITALIDADE E TURISMO FRENTE À

PANDEMIA DE COVID-19: UMA PROPOSTA DE

PESQUISA

capacete. Logo, evidenciou-se que a atitude de ver o uso do capacete como importante ou benéfico teve uma relação muito forte com a intenção de se usar o capacete.

Em outro estudo, desta vez relacionado ao uso de tecnologias no turismo, Mendes-Filho et al. (2018) mostrou que a atitude influencia positivamente a intenção de uso dos conteúdos gerados pelos usuários na Internet durante o planejamento de viagens. Levando isso em consideração, propõe-se aqui a primeira hipótese:

Hipótese 1: A atitude dos turistas em relação às tecnologias RAISA influencia positivamente a intenção de uso das tecnologias RAISA em serviços de hospitalidade das localidades turísticas.

\subsection{Normas Subjetivas (TCP)}

Segundo Ajzen (1991), as normas subjetivas são um fator social que se referem à pressão social percebida pelos indivíduos ou grupos para realizar ou não o comportamento. São as influências causadas pela pressão social de pais, amigos, grupos de referência, cultura, opinião pública e instituições sobre o comportamento dos indivíduos. Assim, acredita-se que as crenças normativas combinadas com a motivação pessoal em agir em conformidade com as expectativas sociais determinam a norma subjetiva que irá prevalecer (PEIXOTO, 2007).

A literatura mostra que a opinião externa impacta a atitude de um indivíduo diretamente, já que muitos indivíduos valorizam a opinião dos outros, o que contribui também para o aprendizado e experiência, uma vez que opiniões externas aumentam a quantidade de informação disponível do tomador de decisão, influenciando assim sua atitude e, consequentemente, seu processo de escolha. Para Mendes-Filho, Tan e Mills (2012), a relação entre as normas subjetivas e as intenções comportamentais têm recebido suporte na literatura, como para Morris e Venkatesh (2000), que analisaram as intenções de adoção e uso contínuo da tecnologia da informação e mostraram que os trabalhadores foram fortemente influenciados pelas normas subjetivas.

Para o estudo de Silveira (2016), opiniões favoráveis de terceiros (geralmente pais e amigos) aumentaram a intenção de se usar o capacete ao andar de bicicleta. Sendo assim, a seguinte hipótese é proposta nesta pesquisa:

Hipótese 2: As normas subjetivas para o uso das tecnologias RAISA influenciam positivamente a intenção de uso dessas tecnologias em serviços de hospitalidade das localidades turísticas.

Revista Acadêmica Observatório de Inovação do Turismo, v. 15, n. 3, dezembro/2021 
ARTIFICIAL E AUTOMAÇÃO DOS SERVIÇOS

EM HOSPITALIDADE E TURISMO FRENTE À

PANDEMIA DE COVID-19: UMA PROPOSTA DE

PESQUISA

\subsection{Expectativa de Desempenho (UTAUT)}

Como primeiro constructo da UTAUT é importante trazer a sua conceituação no sentido de melhor entender a necessidade deste para avaliar a intenção de uso das tecnologias RAISA em serviços de hospitalidade das localidades turísticas. Então, de acordo com Venkatesh et al. (2003) a expectativa de desempenho é definida como o grau em que um indivíduo acredita que, ao utilizar um sistema, este irá ajudá-lo a obter ganhos de performance ou desempenho.

$\mathrm{Na}$ sua criação foram utilizados cinco constructos de outras teorias: utilidade percebida (TAM/TAM2 e C-TAM-TPB), motivação extrínseca (MM), emprego ajustado (MPCU), vantagem relativa (TDI) e resultado esperado (TCS). Fazendo uso das literaturas existentes, foi verificado em um estudo conduzido na Finlândia, por Carlsson et al. (2006), a aplicabilidade do modelo UTAUT frente à aceitação de dispositivos móveis e serviços. Foram utilizados dados de 157 clientes, através de regressão linear, e conseguiram verificar que a expectativa de desempenho tem relação direta com a intenção comportamental.

Os resultados do estudo de Gomes e Farias (2017) sobre o uso de um aplicativo de compras corroboram com a contribuição teórica de Venkatesh, Thong e Xu (2012), quando afirmaram que a Expectativa de Desempenho é o principal condutor da intenção de uso e do uso em si de tecnologias em contextos organizacionais. Contudo, em contextos de consumidores - como é o caso deste estudo de uso das tecnologias RAISA, existem outros fatores que também podem ser condutores da intenção comportamental e uso de uma tecnologia.

Então, é necessária uma atenção especial a essa questão para a realização da pesquisa. Por fim, cabe lembrar que o constructo expectativa de desempenho sofre influência do moderador gênero e idade, o que deve também ser levado em questão. Então, com base nessas afirmações trazidas aqui, faz-se a seguinte proposição de hipótese:

Hipótese 3: A expectativa de desempenho em relação ao uso das tecnologias RAISA influenciam positivamente a intenção de uso dessas tecnologias em serviços de hospitalidade das localidades turísticas. 
ARTIFICIAL E AUTOMAÇÃO DOS SERVIÇOS

EM HOSPITALIDADE E TURISMO FRENTE À

PANDEMIA DE COVID-19: UMA PROPOSTA DE

PESQUISA

\subsection{Expectativa de Esforço (UTAUT)}

Outro constructo considerado no modelo UTAUT é a Expectativa de Esforço, o qual Venkatesh et al. (2003) definiram como o grau de facilidade associada ao uso do sistema. Tal construto da UTAUT se originou a partir de três constructos de diferentes teorias, sendo eles: a) Facilidade de uso percebida, do modelo TAM e TAM2 (DAVIS, 1989; DAVIS et al., 1989); b) Complexidade do modelo MPCU (Thompson et al., 1991); e a c) Facilidade de uso do modelo IDT (MOORE; BENBASAT, 1991).

A expectativa de esforço pode ser também definida como o nível em que um indivíduo percebe que se esforçará ao utilizar determinado sistema ou tecnologia (VENKATESH et al., 2003). E com o aumento do uso das tecnologias, esse tema é um dos que mais chama atenção, uma vez que para Rogers (1995 apud GOMES; FARIAS, 2017), a complexidade é uma das barreiras à inovação. Ou seja, muitos indivíduos não querem nem tentar fazer uso de algumas tecnologias por ser, algumas vezes, algo mais complexo ou necessitar de um aprendizado novo.

Então, para Pikkarainen et al. (2004) a aceitação de um novo sistema ou tecnologia será maior quando o aprendizado sobre sua utilização se mostrar fácil e acessível. Por outro lado, mas corroborando com o que foi dito, Wong et al. (2015) afirmam que quando um sistema é "amigo do usuário", menores serão as barreiras para que os clientes o utilizem. No caso específico do UTAUT, alguns moderadores são propostos para cada constructo como influências. No caso da expectativa de esforço, vale lembrar que gênero, idade e experiência causam influências no mesmo. Posto isto, propõe-se a seguinte hipótese:

Hipótese 4: A expectativa de esforço em relação ao uso das tecnologias RAISA influenciam positivamente a intenção de uso dessas tecnologias em serviços de hospitalidade das localidades turísticas.

\subsection{Condições Facilitadoras (UTAUT)}

Segundo Venkatesh et al. (2003), as condições facilitadoras podem ser definidas como o grau que um indivíduo acredita que existe uma organização ou infraestrutura técnica para apoiar o uso do sistema. Essa definição captura conceitos incorporados por três constructos diferentes: controle comportamental percebido (TPB /DTPB, C-TAM-TPB), condições facilitadoras (MPCU) e

Revista Acadêmica Observatório de Inovação do Turismo, v. 15, n. 3, dezembro/2021 


\section{ARTIFICIAL E AUTOMAÇÃO DOS SERVIÇOS \\ EM HOSPITALIDADE E TURISMO FRENTE À \\ PANDEMIA DE COVID-19: UMA PROPOSTA DE \\ PESQUISA}

compatibilidade (IDT). Assim, cada um desses constructos é operacionalizado para incluir aspectos do ambiente tecnológico e/ou organizacional que são projetados para remover barreiras ao uso.

Ao idealizar a teoria UTAUT, Venkatesh et al. (2003) integraram 32 fatores usados em oito modelos concorrentes em cinco construtos e identificaram empiricamente que a intenção comportamental e as condições facilitadoras eram dois determinantes diretos do comportamento de adoção. No caso específico dessa proposição de estudo, fora do ambiente interno das organizações, acredita-se que a questão das condições facilitadoras de acesso às tecnologias RAISA pode ser vista também como orientação de uso passo a passo, suporte mínimo de pessoal ou até mesmo ações de orientação e educação do cliente para o uso de tais recursos. Além disso, ao fazer uma análise da literatura existente, foi possível observar diversos estudos que traziam as condições facilitadoras como um constructo influente no comportamento.

Um resultado que pode ser citado é o que foi observado no estudo de Yu (2012) sobre os fatores que influenciam as pessoas na adoção do Internet Banking, quando aponta em seus achados que as condições facilitadoras e a intenção comportamental foram encontradas como dois determinantes diretos na influência do comportamento de adoção real das pessoas. Então, faz-se aqui a proposição da seguinte hipótese:

Hipótese 5: A existência de condições facilitadoras em relação ao uso das tecnologias RAISA influenciam positivamente o comportamento de uso dessas tecnologias em serviços de hospitalidade das localidades turísticas.

\subsection{Intenção (TCP e UTAUT)}

De acordo com Ajzen (1991), de maneira similar à TRA, um fator central na TCP é a intenção do indivíduo em realizar um determinado comportamento. Tais intenções são assumidas para capturar os fatores motivacionais que podem assim influenciar um comportamento. São indicações de como as pessoas estão dispostas a tentar, do quanto de esforço estão planejando exercer, a fim de executar o comportamento. Como regra geral, quanto mais forte é a intenção de envolver-se em um comportamento, mais provável deve ser seu desempenho.

E assim, observa-se que tanto a TRA quanto a TCP afirmam que o comportamento é uma função direta da intenção comportamental. Fazendo uso do mesmo constructo, a UTAUT, por sua vez, é

Revista Acadêmica Observatório de Inovação do Turismo, v. 15, n. 3, dezembro/2021 


\section{ARTIFICIAL E AUTOMAÇÃO DOS SERVIÇOS \\ EM HOSPITALIDADE E TURISMO FRENTE À \\ PANDEMIA DE COVID-19: UMA PROPOSTA DE \\ PESQUISA}

consistente com todos os modelos baseados em teorias psicológicas, trazendo a argumentação de que o comportamento individual é previsível e influenciado pela intenção individual (VENKATESH et al. 2003).

E ainda, de acordo com Ajzen (1991), a intenção é peça-chave no direcionamento do comportamento, além de ser responsável por capturar os fatores motivacionais que influenciam um comportamento. Portanto, a intenção indica como as pessoas estão dispostas a tentar, ou seja, quanto de esforço estão planejando exercer, a fim de realizar o comportamento. Desse modo, faz-se aqui a proposição da última hipótese:

Hipótese 6: A intenção de uso das tecnologias RAISA influencia positivamente o comportamento de uso dessas tecnologias em serviços de hospitalidade das localidades turísticas.

\section{Considerações Finais}

Partindo do pressuposto básico do aumento do acesso e, consequentemente, do uso das tecnologias nos diversos setores do mercado e no cotidiano das pessoas, - mudanças essas que ainda foram impulsionadas no último ano de 2020 principalmente pelas necessidades nitidamente impostas pelo surgimento da pandemia da COVID-19, a qual exigiu um isolamento social - inúmeras transformações surgiram na relação de compra e venda no mercado. Somado a tudo isso, há ainda a preocupação das empresas em fornecer uma experiência de qualidade, que seja inovadora e diferenciada e que não apenas venda um simples produto ou serviço.

Desta maneira, surge o interesse de fornecer argumentos para a realização de uma pesquisa futura sobre o uso e aceitação das tecnologias RAISA pelos turistas em destinações visitadas, tendo esse artigo teórico por objetivo principal desenvolver um novo modelo com a integração das teorias TCP e UTAUT, de modo a compreender a possibilidade de uso e aceitação das tecnologias RAISA pelos turistas em serviços de hospitalidade das localidades turísticas. Conforme mencionado, vale ressaltar que a pandemia de COVID-19 ampliou o uso das tecnologias, em virtude da necessidade de distanciamento social, protocolos de higiene e restrições às viagens.

Existe a intenção de sugerir a realização de uma pesquisa empírica futura acerca do uso e aceitação das tecnologias RAISA pelos turistas em serviços de hospitalidade das localidades turísticas, que testem as hipóteses aqui levantadas, ou seja, até que ponto a atitude, as normas subjetivas, a expectativa de desempenho e a expectativa de esforço em relação ao uso das tecnologias RAISA influenciam positivamente a intenção de uso. Além disso, deve-se considerar também, até que ponto as

Revista Acadêmica Observatório de Inovação do Turismo, v. 15, n. 3, dezembro/2021 
ARTIFICIAL E AUTOMAÇÃO DOS SERVIÇOS

EM HOSPITALIDADE E TURISMO FRENTE À

PANDEMIA DE COVID-19: UMA PROPOSTA DE

PESQUISA

condições facilitadoras e a intenção influenciam positivamente o comportamento de uso dessas tecnologias em serviços de hospitalidade das localidades turísticas.

Neste estudo, os constructos foram escolhidos por se adequarem ao objetivo de analisar o comportamento dos turistas nas destinações que possuam ou queiram desenvolver as tecnologias RAISA e sua intenção de uso. Assim, analisando a Atitude, Normas Subjetivas, Expectativa de Desempenho, Expectativa de Esforço, Condições Facilitadoras, Intenção e Comportamento de Uso, acredita-se que seja possível ter um melhor entendimento sobre a existência de recursos tecnológicos que podem influenciar a intenção de comportamento de compra e uso de tais tecnologias pelos turistas.

Com isso, são inúmeros os benefícios para o setor de hospitalidade e turismo, ao ser possível avaliar a predisposição dos turistas em utilizar recursos tecnológicos em suas próximas viagens, gerando, assim, dados e informações úteis para os diversos agentes da cadeia produtiva de turismo. Estes, por sua vez, poderão moldar seus produtos e serviços dentro dessas novas características. Portanto, tais informações são extremamente relevantes, principalmente pelo fato de o mercado estar hoje muito mais voltado a proporcionar boas experiências para os consumidores, de forma a entender o seu comportamento e o que o motiva a tomar sua decisão de compra, oferecendo o melhor serviço e melhorando a competitividade do destino.

Uma limitação do presente estudo é o fato do mesmo ser teórico-conceitual, assim, sugere-se a realização não apenas da pesquisa proposta com o modelo aqui elaborado, mas com a execução de pesquisas futuras com abordagens quantitativas, as quais possam até mesmo avaliar se esse modelo de fato consegue responder às necessidades relatadas sobre entender o comportamento de tais consumidores em destinações turísticas.

Não se pretende esgotar nesse estudo toda a complexidade dos temas tratados. Pelo contrário, faz-se necessária a criação de novos modelos, com o uso da integração de outras teorias e a intenção de investigar a temática mais a fundo, de modo a servir de base para a condução de outros estudos sobre o tema.

\section{Referências}

AJZEN, Icek. The Theory of Planned Behavior. Organizational Behavior and Human Decision Processes. University of Massachusetts at Amherst, v. 50, n. 2, p. 179-211, 1991. 
ARMITAGE, C. J.; CHRISTIAN, J. From Attitudes to Behavior: Basic and Applied Research on the Theory of Planned Behavior. Current Psychology, v. 22, n. 3, p. 187-195, 2003.

BOBSIN, Débora; VISENTINI, Monize Sâmara; RECH, Ionara. Em busca do estado da arte do UTAUT. Revista de Administração e Inovação, v. 6, n. 2, 2009.

BOTELHO, Laís Vargas; CARDOSO, Letícia de O.; CANELLA, Daniela S. COVID-19 e ambiente alimentar digital no Brasil: reflexões sobre a influência da pandemia no uso de aplicativos de delivery de comida. Cadernos de Saúde Pública, v. 36, n. 11, 2020.

CARLSSON, C.; CARLSSON, J.; HYVONEN, K.; PUHAKAINEN, J.; WALDEN, P. Adoption of mobile devices/services-searching for answers with the UTAUT. Anais da 39th Annual Hawaii International Conference on System Sciences (HICSS'06), 132a, 2006.

DAVIS, F. D., BAGOZZI, R. P., WARSHAW, P. R. User acceptance of computer technology: a comparison of two theoretical models. Management Science, v. 35, n. 8, p. 982-1003, 1989.

GALANAKIS, Charis M., RIZOU, Myrto, ALDAWOUD, Turki M.S., UCAK, Ilknur; ROWAN Neil J. Innovations and Technology Disruptions in The Food Sector Within the Covid-19 Pandemic and PostLockdown Era. Trends In Food Science \& Technology, v. 110, p. 193-200, 2021.

GOMES, C. M. R.; FARIAS, J. S. A influência da Expectativa de Desempenho e de Esforço Percebidas por Usuários no Uso de um Aplicativo de Compras. Contabilidade, Gestão e Governança, ANPAD. v.20, n.1, p.72-90, 2017.

HALL, C. Michael; SCOTT, Daniel; GOSSLING, Stefan. Pandemics, transformations and tourism: be careful what you wish for. Tourism Geographies. v. 22, n. 3, p. 577-598, 2020.

HEINEKE, K.; KAMPSHOFF, P.; MKRTCHYAN, A.; SHAO, E. Self-driving car technology: When will the robots hit the road? McKinsey \& Company. Disponível em https://www.mckinsey.com/industries/automotive-and-assembly/our-insights/self-driving-cartechnology-when-will-the-robots-hit-the-road. Acesso em 17 mai. 2021.

IVANOV, S.; GRETZEL, Ulrike; BEREZINA, Katerina; SIGALA, Marianna; WEBSTER, Craig. Progress on robotics in hospitality and tourism: a review of the literature. Journal of Hospitality and Tourism Technology. v. 10, n. 4, p. 489-521, 2019.

IVANOV, S.; WEBSTER, C. Adoption of Robots, Artificial Intelligence and Service Automation by Travel, Tourism and Hospitality Companies - A Cost-benefit Analysis. Anais da International 
ARTIFICIAL E AUTOMAÇÃO DOS SERVIÇOS

EM HOSPITALIDADE E TURISMO FRENTE À

PANDEMIA DE COVID-19: UMA PROPOSTA DE

PESQUISA

Scientific Conference "Contemporary tourism - traditions and innovations". Sofia University, p. 19-21, 2017.

IVANOV, S.; WEBSTER, C. Robots in tourism: a research agenda for tourism economics. Tourism Economics (forthcoming). Invited Commentary Article, 2020.

MATOS, M. G.; SARDINHA, L. B. Estilos de vida activos e qualidade de vida, Lisboa: Edições FMH, 1999.

MENDES-FILHO, L., MILLS, A. M., TAN, F. B.; MILNE, S. Empowering the traveler: an examination of the impact of user-generated content on travel planning, Journal of Travel \& Tourism Marketing, v. 35, n. 4 , p. 425-436, 2018.

MENDES-FILHO, Luiz; TAN, Felix; MILLS, Annette. User-generated content and travel planning: An application of the theory of planned behavior. Revista Brasileira de Pesquisa em Turismo, v.6, n.3, p. 20-29, 2012.

MEUTER, M. L.; OSTROM, Amy; ROUNDTREE R.; BITNER M. J. Self-Service Technologies: Understanding Customer Satisfaction with Technology-Based Service Encounters. Journal of Marketing, v.64, n.3, p.50-64, 2000.

PARVEZA, M. O.; COBANOGLU, C. Opportunities and Challenges of Utilizing Service Robots in Tourism Industry: A Tool for Recovery from COVID-19 Pandemic. Journal of Smart Tourism, v.1 n.3, p. 17-20, 2021.

PEIXOTO, F.C. Estudo do comportamento planejado na escolha da faculdade: uma aplicação ao contexto itabirano. Dissertação de Mestrado em Administração. FUMEC. Belo Horizonte, MG, 2007.

PIKKARAINEN, Tero; PIKKARAINEN, Kari; KARJALUOTO, Heikki; PAHNILA, Seppo. Consumer acceptance of online banking: An extension of the Technology Acceptance Model. Emerald Publishing, v.14, n.3, 2004.

PINE, Joseph; GILMORE, James H. Bem-vindo à economia da experiência. Harvard Business Review, 1998. Disponível em < https://hbr.org/1998/07/welcome-to-the-experience-economy>. Acesso em 06 dez. 2021.

PINTO, Marcelo de Rezende. A Teoria do Comportamento Planejado (TCP) e o índice de disposição de adoção de produtos e serviços baseados em tecnologia (TRI): uma interface possível? Revista Gestão \& Tecnologia, v.7, n.2, p. 1-13, 2007. 
RODRIGUES, A; ASSMAR, E. M. L.; JABLONSKI, B. Psicologia Social. 18. ed. Rio de Janeiro: Vozes, 2000.

SILVEIRA, Mariana Oliveira. O uso da bicicleta sob os fundamentos da teoria do comportamento planejado. Tese. UFPE, 2016.

VENKATESH, Viswanath; MORRIS, Michael; DAVIS, Gordon; DAVIS, Fred D. User acceptance of information technology: toward a unified view. MIS Quarterly. v.27, n.3, p. 425-478, 2003.

VENKATESH, Viswanath; THONG, James Y. L.; XU, Xin. Consumer Acceptance and Use of Information Technology: Extending the Unified Theory of Acceptance and Use of Technology. MIS Quarterly. v.36, n.1, p. 157-178, 2012.

WILLEMS, K., smolders, A., BRENGMAN, M., LUYTEN, K.; SCHONING, J. The path to purchase is paved with digital opportunities: An inventory of shopper-oriented retail technologies.

Technological Forecasting \& Social Change. An International Journal. v.124, n.C, p. 228-242, 2017.

WONG, C. H., TAN, G. W. H., TAN, B. I.; OOI, K. B. Mobile advertising: the changing landscape of the advertising industry. Telematics and Informatics, v.32, n.4, p.720-734, 2015.

YU, Chian-Son. Factors affecting individuals to adopt mobile banking: empirical evidence from the UTAUT model. Journal of Electronic Commerce Research. v.13, n.2, p.104-121, 2012.

ZENG, Zhanjing; CHEN, Po-Ju; LEW, Alan A. From high-touch to high-tech: COVID-19 drives robotics adoption. Tourism Geographies, v.22, n.3, p. 724-734, 2020. 